\title{
Telomere length variation in biliary tract metaplasia, dysplasia, and carcinoma
}

\author{
Donna E Hansel ${ }^{1}$, Alan K Meeker ${ }^{1,2}$, Jessica Hicks², Angelo M De Marzo², \\ Keith D Lillemoe ${ }^{3, *}$, Richard Schulick ${ }^{3}$, Ralph H Hruban ${ }^{1}$, Anirban Maitra ${ }^{1}$ \\ and Pedram Argani ${ }^{1}$
}

${ }^{1}$ Department of Pathology, The Johns Hopkins Medical Institutions, Baltimore, MD, USA; ${ }^{2}$ Brady Urological Institute, The Johns Hopkins Medical Institutions, Baltimore, MD, USA and ${ }^{3}$ Department of Surgery, The Johns Hopkins Medical Institutions, Baltimore, MD, USA

\begin{abstract}
Biliary tract carcinoma, including carcinoma of the gallbladder, intrahepatic bile ducts (cholangiocarcinoma), and extrahepatic bile ducts, affects 7500 people in the United States annually, and has an overall 32\% 5-year survival rate for disease limited to the mucosa, and a dismal 10\% 5-year survival for more advanced disease. The identification of factors involved in the pathogenesis and progression of biliary tract carcinoma is critical for devising effective methods of screening and treatment. Recent evidence suggests that reduction of the length of telomeres, which normally help maintain chromosomal stability, may promote the development and progression of a variety of carcinomas. Using a novel, recently validated telomere fluorescence in situ hybridization method, we examined telomere length in normal and inflamed gallbladder epithelium, metaplasia and dysplasia of the gallbladder, and biliary tract carcinoma to determine whether telomere shortening is associated with neoplastic progression in the biliary tract. Although normal and inflamed gallbladder epithelium demonstrated uniform normal telomere lengths, over half of all metaplastic lesions demonstrated shortened telomeres, supporting prior evidence that metaplastic lesions of the gallbladder are pre-neoplastic. Dysplastic epithelium and invasive carcinomas demonstrated almost universally abnormally short telomeres, indicating that telomere shortening occurs at an early, preinvasive stage of cancer development. In addition, invasive adenocarcinoma of the biliary tract frequently demonstrated intratumoral heterogeneity of telomere lengths. We conclude that telomere shortening is a consistent and early finding in the development of biliary tract carcinoma.
\end{abstract}

Modern Pathology (2006) 19, 772-779. doi:10.1038/modpathol.3800591; published online 24 March 2006

Keywords: telomere; gallbladder; bile duct; carcinoma; dysplasia; metaplasia

Biliary tract carcinoma arises within the biliary epithelium of the gallbladder, extrahepatic bile ducts, and smaller intrahepatic bile ducts. ${ }^{1-3}$ Dysplastic changes of this epithelium, including flat in situ carcinoma, are recognized as precursor lesions for invasive biliary tract carcinoma. Additionally, metaplastic changes have been implicated as an even earlier potential precursor in gallbladder carcinoma. ${ }^{4,5}$ Risk factors for the development of biliary tract carcinoma include race (increased risk in native and Hispanic Americans), cholelithiasis, abnormal junction of the pancreatic and biliary duct for gallbladder carcinoma, and primary sclerosing

Correspondence: Dr P Argani, MD, Department of Pathology, The Johns Hopkins University School of Medicine, 401 N Broadway, Weinberg 2242, Baltimore, MD 21231-2410, USA.

E-mail: pargani@jhmi.edu

${ }^{*}$ Current address: Indiana University School of Medicine, Indianapolis, IN, USA.

Received 19 January 2006; revised 22 February 2006; accepted 23 February 2006; published online 24 March 2006 cholangitis and choledochal cyst for carcinoma of the extrahepatic bile ducts. The frequent advanced nature of disease on presentation, as well as limited knowledge of causative molecular factors underlying biliary tract carcinoma, has limited the therapeutic options for patients with this cancer. Identification of early molecular changes in biliary cancer could provide insight into key genetic events that could aid in the development of screening and targeted treatment strategies for biliary tract carcinoma.

Previous work on biliary tract carcinoma has identified a number of molecular abnormalities, including mutations in KRAS, ${ }^{6}$ CDKN2A/TP16, ${ }^{7}$ $T P 53,{ }^{8}$ and $A P C .{ }^{9}$ Recent evidence from a variety of other cancer models has indicated that telomere shortening may also contribute to carcinoma progression, although this has not been previously examined in biliary tract carcinoma. ${ }^{10-12}$ For example, telomere shortening appears to be an early and common event in precursors to invasive prostatic and pancreatic cancer. ${ }^{13,14}$ 
Telomeres consist of DNA-protein structures, which serve as 'caps' on the ends of linear chromosomes that allow cells to distinguish between double-stranded breaks in the DNA and normal chromosome ends. ${ }^{15,16}$ In humans, telomeric DNA sequences consist of 1000-2000 tandem repeats of TTAGGG, which generally shorten following cell division owing to incomplete replication of telomere repeats during DNA synthesis (the 'end-replication problem'). Severe telomere shortening can result in degradation of proximal or internal DNA with subsequent loss of genetic information, recombination of DNA at these sites, or fusion of chromosomes containing shortened telomeres. ${ }^{17,18}$ The appropriate outcomes of telomere shortening in somatic cells are thought to be cell senescence or apoptosis, which decreases the population of dividing, genetically unstable cells. Expression of telomerase, the enzyme that catalyzes the extension of telomeres, is found in $85-90 \%$ of carcinomas ${ }^{19,20}$ and may serve to stabilize chromosomes with short telomeres, thus leading to a third possible outcome: immortalization of genetically unstable cancer cells.

In the present study, using a novel in situ method, we examined telomere lengths in normal and inflamed gallbladder mucosa, metaplasia and dysplasia of the gallbladder, and invasive biliary tract carcinoma to understand the role of telomere shortening in different stages of biliary carcinoma progression. We report here the identification of telomere shortening in metaplastic lesions, dysplastic lesions, and invasive biliary tract carcinoma.

\section{Materials and methods}

\section{Patients and Tissue Samples}

This study was approved by The Johns Hopkins Hospital Institutional Review Board. All tissue samples studied were derived from formalin-fixed, paraffin-embedded tissue blocks. The majority of invasive biliary tract carcinomas studied were derived from a biliary carcinoma tissue microarray constructed at The Johns Hopkins Hospital. This tissue microarray consists of $2 \mathrm{~mm}$ diameter spots of tumor derived from 13 gallbladder carcinomas, 10 intrahepatic cholangiocarcinomas, and 13 distal common bile duct carcinomas, along with five normal gallbladder spots. Additional whole tissue sections were obtained from the following lesions, which were not represented on the tissue microarray: cholecystitis (12 cases, five demonstrating metaplasia and seven not demonstrating metaplasia), dysplasia unaccompanied by invasive carcinoma (three cases, one low-grade dysplasia and two high-grade dysplasia/carcinoma in situ), carcinoma in situ and invasive carcinoma of the gallbladder (eight cases), and small cell carcinoma of the gallbladder (two cases, one of which contained a component of adenocarcinoma). Four additional whole sections of invasive gallbladder adenocarci- noma were also examined. Specimens were obtained through surgical resection for a variety of conditions, including cholelithiasis, cholecystitis, and biliary tract carcinoma. In total, 65 patient specimens were obtained for study, including specimens demonstrating multiple abnormalities (ie, dysplasia and invasive carcinoma). Average patient age was 60 years. Overall, $39 \%$ of patients were male, and $61 \%$ of patients were female. Male and female ratios were similar for all specimen diagnosis groups. Tissue sections (3-4 $\mu \mathrm{m}$ ) from each formalinfixed, paraffin-embedded tissue block were utilized for the staining protocol (see below).

\section{Combined Telomere Fluorescence In Situ Hybridization}

Paraffin slides were processed and labeled as described previously, with the omission of protease treatment. ${ }^{12}$ Briefly, slides were deparaffinized and rehydrated through a series of ethanol gradients. Slides were then treated with $0.1 \%$ Tween 20 detergent in deionized water and incubated in citrate buffer (Vector Laboratories, Burlingame, CA, USA) in steam (Black and Decker Vegetable Steamer, Towson, MD, USA), washed in PBS with Tween, placed briefly in $95 \%$ ethanol, and air dried. A $25 \mu \mathrm{l}$ portion of Cy3-labeled telomere-specific peptide nucleic acid (PNA; $0.3 \mu \mathrm{g} / \mathrm{ml}$ PNA in $70 \%$ formamide, $10 \mathrm{mmol} / \mathrm{l}$ Tris, $\mathrm{pH}$ 7.5, 0.5\% B/M Blocking reagent; Boehringer-Mannheim, Indianapolis, IN, USA) was added to each slide. Slides were then coverslipped, incubated for $4 \mathrm{~min}$ at $83^{\circ} \mathrm{C}$, and hybridized for $2 \mathrm{~h}$ at room temperature in the dark. Slides were then washed twice in PNA wash solution $(70 \%$ formamide, $10 \mathrm{mmol} / \mathrm{l}$ Tris, $\mathrm{pH} 7.5$, $0.1 \%$ albumin; Sigma, St Louis, MO, USA), followed by three PBS washes. Slides were counterstained with 4'-6-diamidino-2-phenylindole in deionized water (DAPI; Sigma Chemical Company) and mounted with Prolong anti-fade mounting medium (Molecular Probes Inc., Eugene, OR, USA). Serial sections were stained using hematoxylin and eosin and used as a morphology reference during telomere analysis.

\section{Microscopy}

Slides were imaged with a Zeiss Axioskop epifluorescence microscope equipped with short-arc mercury lamp illumination (Carl Zeiss Inc., Thornwood, NY, USA) and a $\times 100 / 1.4$ NA oil immersion Neofluar lens. Fluorescence excitation/emission filters were Cy3 546/10 nm BP excitation, $578 \mathrm{~nm} \mathrm{LP}$ emission (Carl Zeiss Inc.) and DAPI $330 \mathrm{~nm}$ excitation and $400 \mathrm{~nm}$ emission via an XF02 fluorescence set (Omega Optical, Brattleboro, VT, USA). Fluorescent images were captured with a cooled charge-coupled device camera (Micro MAX digital camera, Princeton Instruments, Trenton, NJ, USA). 


\section{Image and Data Analysis}

Five regions of each tissue section were examined for telomere labeling, and intensity of fluorescence was utilized as a reflection of telomere length based upon prior validation of this technique. ${ }^{12}$ A subset of these cases were further quantified using a method in which the sum of pixel intensities in the Cy3 channel for a given cell nucleus is normalized to the DAPI signal as described previously. ${ }^{14}$ The telomere fluorescence in situ hybridization (FISH) intensity of inflamed, metaplastic, dysplastic, and carcinomatous epithelium was compared to the corresponding normal epithelium on the same section, if present, or, if absent, to adjacent stroma. Normal epithelium, endothelium, and stromal cells demonstrated equal intensity of telomere staining in all samples analyzed. Lymphocytes in all cases demonstrated intensely fluorescent telomeres relative to epithelial and stromal cells, consistent with prior observations that lymphocytes have elongated telomeres. Telomere fluorescence intensity was scored visually by three observers (DEH, AKM, PA) as increased (long), equal (normal), or decreased (short) relative to normal epithelium or stroma. In cases in which different areas of a tumor had slightly differing telomere intensities, such that the intensities ranged from short to normal or normal to long, tumors were scored on the basis of the predominant (most frequently observed) telomere intensity seen but also noted to be slightly heterogeneous. Cases in which marked variability of intensity of telomere labeling was noted within a tumor, such that the intensity ranged from normal to virtually inapparent or increased to decreased, were classified as markedly heterogeneous. Cases in which normal stroma did not yield a detectable signal or those in which background autofluorescence obscured telomere signals (presumably owing to fixation artifacts) were excluded from the study. Additional quantification of telomere lengths in three cases of gallbladder metaplasia and two cases of carcinoma in situ was performed. An average of 19 representative nuclei per epithelial cell subtype (normal, metaplastic, and carcinoma in situ) were quantified. For each patient sample, comparisons of the mean ratios of telomeric signal to DAPI between the various cell types were made using the paired $t$-test.

\section{Results}

\section{Normal Gallbladder Epithelium Demonstrates Uniform Telomere Lengths}

All five of the normal gallbladder specimens examined demonstrated uniform, bright telomere signals in all epithelial cells (Figure 1a). The telomere signal intensity of the normal epithelium appeared equivalent to that of stromal cells, but appreciably less than that of lymphocytes, which consistently displayed bright telomere staining.
Acute and Chronic Inflammation of the Gallbladder Does not Alter Telomere Length

As dysplasia and carcinoma of the biliary tract has been proposed to arise in the setting of chronic inflammation with increased epithelial cell turnover, ${ }^{21}$ we examined telomere labeling in inflamed, reactive gallbladder epithelium. We examined seven specimens with acute, chronic, or mixed acute and chronic inflammation of the biliary epithelium. Two of these specimens demonstrated florid acute cholecystitis with abscess formation. In all cases $(100 \%)$, telomere length was identical between normal epithelium, stroma, and inflamed epithelium (Figure 1b).

\section{Telomere Shortening is Evident in a Subset of Metaplastic Lesions of the Gallbladder}

Metaplastic epithelial changes occur following ongoing injury to the normal, columnar, absorptivetype biliary epithelium, with resultant formation of gastric or intestinal-type mucinous glands. We therefore examined eight gallbladder specimens demonstrating a prominent region of pyloric or intestinal metaplasia; all specimens had either adjacent cholecystitis (5) or dysplasia (3). Of the eight metaplastic lesions examined, three of the five associated with cholecystitis demonstrated telomere lengths equal to the underlying stroma or adjacent normal epithelium; these included two cases with only pyloric metplasia and one case with both pyloric and intestinal metaplasia. However, the other two examples of cholecystitis with pyloric metaplasia demonstrated focal shortening of telomeres. In all three cases of intestinal metaplasia associated with dysplasia, focal shortening of telomeres was observed (Figure 1c).

\section{Biliary Tract Dysplasia and Carcinoma In Situ Demonstrate Shortened Telomeres}

We next examined three specimens with dysplasia unassociated with invasive carcinoma, and eight specimens with high-grade dysplasia (carcinoma in situ) in the same section as invasive adenocarcinoma. Ten of the $11(91 \%)$ showed shortened telomeres. Among the three cases of dysplasia alone, one case of carcinoma in situ demonstrated elongated telomeres, whereas one case of low-grade dysplasia and one other case of carcinoma in situ demonstrated short telomeres. All eight examples of carcinoma in situ associated with invasive carcinoma demonstrated marked reduction in telomere intensity (Figure 1d). Two cases demonstrated regions of marked heterogeneity of telomere length, with nuclei demonstrating both short and long telomere intensities. In regions where a direct transition between dysplastic epithelium and normal epithelium was present, a dramatic reduction in telomere staining intensity was apparent in the dysplastic epithelium as compared to both normal epithelium and stroma. 

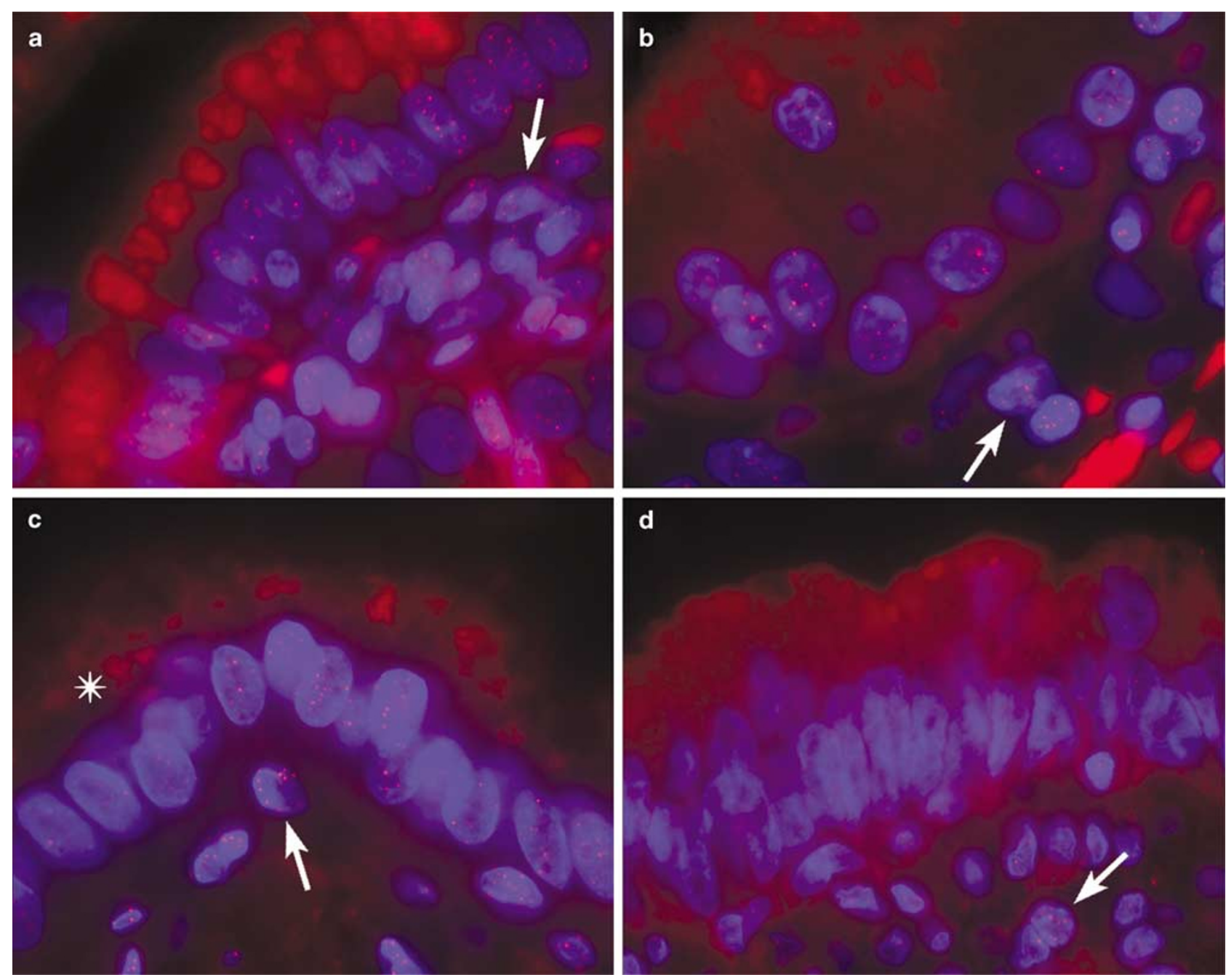

Figure 1 Telomere fluorescence (red dots within blue nuclei). Arrows represent underlying stroma as a comparison. (a) Equal telomere staining intensity between normal biliary epithelium and underlying stroma. (b) Biliary epithelium in acute cholecystitis does not reveal telomere shortening. Note the more rounded nuclei of reactive biliary epithelium, compared to the more elongated normal nuclei in (a). (c) Focal reduced telomere length $(*)$ in metaplastic epithelium. (d) Reduced telomere length in flat in situ carcinoma of the gallbladder. Note the virtual absence of signal in stratified, enlarged, elongated nuclei. Magnification $\times 400$, oil immersion.

\section{Adenocarcinoma of the Biliary Tract Displays Consistently Shortened Telomeres with Intratumoral Cell-Cell Heterogeneity}

We next examined 48 specimens with invasive adenocarcinoma of the gallbladder (25 specimens), intrahepatic bile ducts (10 specimens), or distal common bile duct (13 specimens). Fourty-one of the 48 cases $(85 \%)$ showed shortened telomere lengths. Of the 25 gallbladder carcinomas, 21 demonstrated diminished telomere fluorescence intensity compared to the stroma, although slight cell-cell heterogeneity was noted in five of these cases. The remaining four tumors demonstrated marked cell-cell heterogeneity in telomere length. Of the 10 intrahepatic cholangiocarcinomas, nine demonstrated diminished telomere fluorescence intensity, with slight cell-cell heterogeneity noted in one of these cases. The final case showed marked cell-cell heterogeneity in telomere intensity. Of 13 distal bile duct carcinomas, 11 demonstrated diminished telomere intensity, with four of these 11 showing slight cell-cell heterogeneity in length. One other tumor demonstrated normal telomere intensity, whereas one demonstrated marked cellcell heterogeneity in intensity.

Hence, virtually all adenocarcinoma specimens, regardless of the location of origin within the biliary tract, demonstrated markedly shortened telomeres, as compared to normal regions within the same specimen. These telomeres were frequently undetectable, indicating that the telomeres had shortened beyond the level of sensitivity of our FISH probe (Figure 2a). A striking difference in telomere FISH intensity was apparent between normal epithelium and carcinoma in cases where carcinoma was colonizing overlying normal epithelium (Figure 2b). 

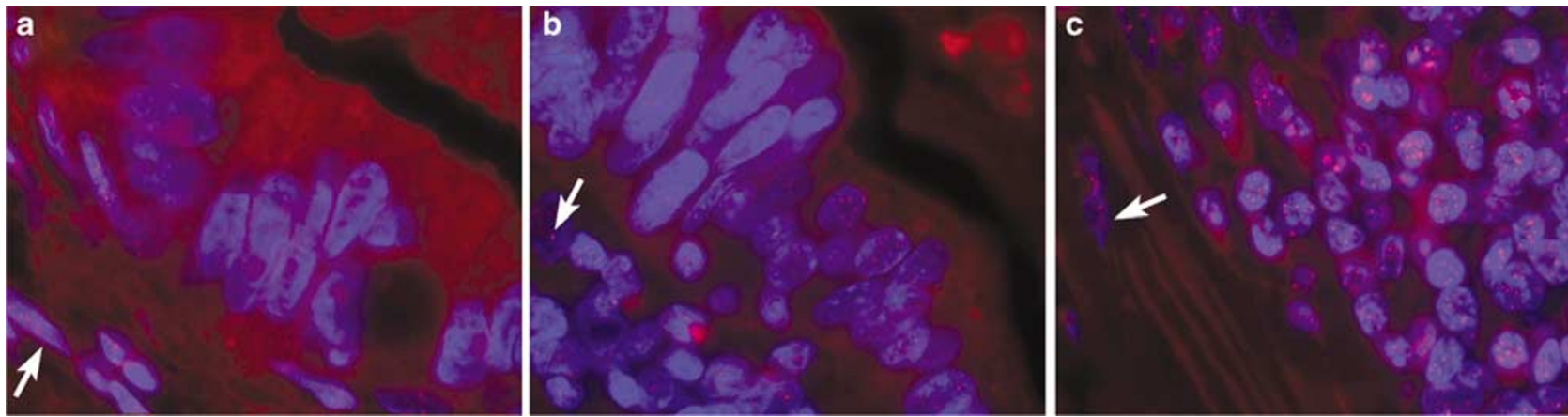

Figure 2 Telomere length variation in a case of combined invasive adenocarcinoma and small cell carcinoma. Arrows represent underlying stroma. (a) Invasive adenocarcinoma demonstrates markedly shortened telomeres. (b) Adenocarcinoma (left upper) colonizing normal epithelium. (c) Small cell carcinoma component associated with this invasive adenocarcinoma demonstrating increased telomere length (more intense red signal) compared to the normal tissue or adenocarcinoma shown in (a) or (b). Magnification $\times 400$, oil immersion.

Table 1 Normalized telomere fluorescence: normal, metaplastic, and dysplastic epithelium unassociated with invasive carcinoma

\begin{tabular}{|c|c|c|c|c|c|c|c|c|}
\hline \multirow[t]{2}{*}{ Lesion } & \multirow[t]{2}{*}{ Telomere intensity } & \multicolumn{3}{|c|}{ Normal epithelium } & \multicolumn{3}{|c|}{ Metaplastic/dysplastic epithelium } & \multirow[t]{2}{*}{$\mathrm{P}$} \\
\hline & & Mean & s.d. & $\mathrm{n}$ & Mean & s.d. & $\mathrm{n}$ & \\
\hline PM in inflam & Short & 9.0 & 2.6 & 19 & 4.1 & 3.0 & 23 & 0.000001 \\
\hline IM in LGD & Short & 2.4 & 1.1 & 26 & 1.0 & 1.0 & 20 & 0.00018 \\
\hline IM in CIS & Short & 3.1 & 2.5 & 18 & 1.6 & 1.0 & 10 & 0.03124 \\
\hline CIS & Short & 5.5 & 4.1 & 12 & 3.5 & 2.3 & 20 & 0.13861 \\
\hline \multirow[t]{2}{*}{ CIS/hetero } & Short & 12.7 & 5.9 & 22 & 1.6 & 1.1 & 18 & 0.00000001 \\
\hline & Long & 12.7 & 5.9 & 22 & 18.5 & 7.8 & 14 & 0.01048 \\
\hline
\end{tabular}

Inflam, inflammation; IM, intestinal metaplasia; PM, pyloric metaplasia; LGD, low-grade dysplasia; CIS/hetero, carcinoma in situ with heterogeneous telomere lengths.

\section{Small Cell Carcinoma Specimens}

In one specimen that contained both small cell carcinoma and adenocarcinoma of the gallbladder, abnormally lengthened telomeres were apparent only within the small cell carcinoma component, whereas the adenocarcinoma component demonstrated abnormally short telomeres (Figure 2c). An additional small cell carcinoma specimen demonstrated shortened telomeres.

\section{Quantification of Telomere Lengths}

To verify the above findings, we performed telomere quantification to correlate telomere intensity with telomere length, as has been described previously. ${ }^{14}$ Three cases of metaplasia were analyzed, one in association with cholecystitis alone, one in association with both cholecystitis and low-grade dysplasia, and one in association with cholecystitis and carcinoma in situ. In addition, one case of carcinoma in situ with reduced telomere intensity and one case of carcinoma in situ with heterogeneous telomere shortening were examined. The results of telomere quantification mirrored the telomere intensities as scored visually (Table 1). In the cases of metaplasia, statistically significant reduced telomere intensity was identified in association with inflammation and dysplasia $(P<0.05)$. In addition, the case of carcinoma in situ with heterogeneous telomere shortening demonstrated a statistically significant difference from normal telomere lengths in both the shortened and elongated telomeres $(P<0.01)$. Finally, another case of carcinoma in situ demonstrated shortened telomeres by quantification, although the results were not statistically significant. A diagram of quantified telomere lengths is presented in Figure 3.

The results of this study are summarized in Table 2 .

\section{Discussion}

Little is known regarding the molecular changes underlying the development of biliary tract carcinomas. ${ }^{1,3}$ In mouse models, dysfunctional telomeres have been shown to induce chromosome instability, leading to chromosomal deletions, amplifications, and rearrangements of the type often found in human biliary carcinomas. ${ }^{10,11,22-25}$ We therefore examined telomere length in biliary tract carcinomas, as well as in inflammation, metaplasia, and dysplasia of the gallbladder, in order to identify potential molecular changes in telomeres in biliary cancer and biliary cancer precursor lesions. We identified shortened telomeres in metaplastic 

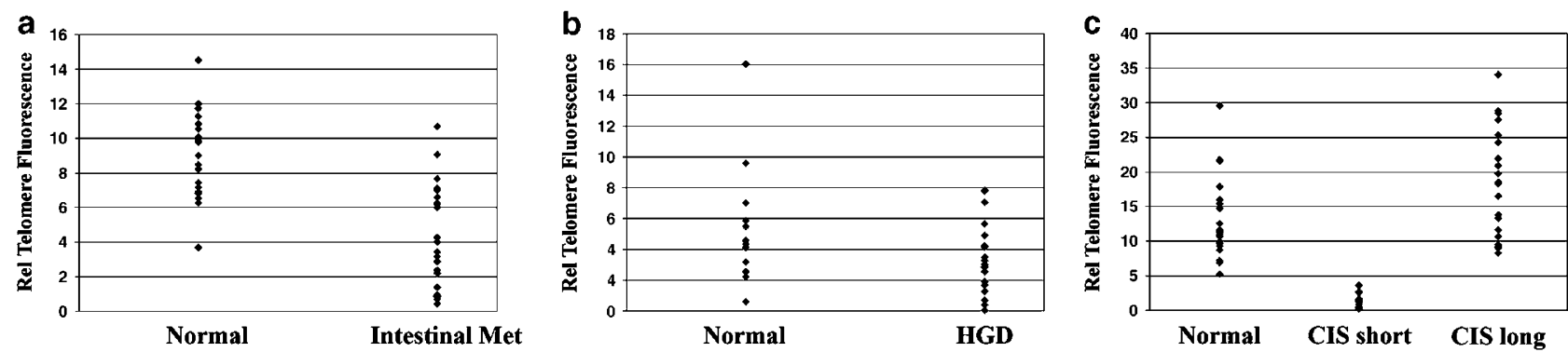

Figure 3 Relative telomere fluorescence in cases of (a) intestinal metasplasia associated with inflammation, (b) high-grade dysplasia/ carcinoma in situ with shortened telomeres, and (c) carcinoma in situ with heterogeneous telomere lengths. Each dot represents a single nucleus.

Table 2 Telomere length assessment: summary of results

\begin{tabular}{|c|c|c|c|c|c|c|c|c|}
\hline \multirow[t]{2}{*}{ Telomere length } & \multirow{2}{*}{$\begin{array}{c}\text { Normal } \\
\text { gallbladder }\end{array}$} & \multirow[t]{2}{*}{ Cholecystitis } & \multicolumn{2}{|c|}{ Metaplasia } & \multicolumn{2}{|c|}{ Dysplasia/CIS } & \multirow{2}{*}{$\begin{array}{c}\text { Invasive } \\
\text { adenocarcinoma }\end{array}$} & \multirow{2}{*}{$\begin{array}{l}\text { Small cell } \\
\text { carcinome }\end{array}$} \\
\hline & & & $\begin{array}{c}\text { In } \\
\text { cholecytitis }\end{array}$ & $\begin{array}{c}\text { In } \\
\text { dysplasia }\end{array}$ & Alone & $\begin{array}{l}\text { With } \\
\text { invasive }\end{array}$ & & \\
\hline Long & & & & & $1 / 3$ & & & $1 / 2$ \\
\hline Normal & $5 / 5$ & $7 / 7$ & $3 / 5$ & & & & $1 / 48$ & \\
\hline Short & & & & & $2 / 3$ & $8 / 8$ & $31 / 48$ & $1 / 2$ \\
\hline Short with heterogeneity & & & $2 / 5$ & $3 / 3$ & & & $10 / 48$ & \\
\hline Marked heterogeneity & & & & & & & $6 / 48$ & \\
\hline
\end{tabular}

epithelium (63\%) and dysplastic epithelium (90\%) of the gallbladder, and infiltrating adenocarcinoma of the gallbladder, intrahepatic bile ducts (cholangiocarcinoma), and extrahepatic bile ducts (98\%).

Telomere shortening may occur in somatic cells following oxidative stress $^{26}$ or repeated cell division, in which incomplete replication of telomere repeats during DNA synthesis reduces telomere length at each division. ${ }^{16}$ In certain cells, such as germline embryonic cells, maintenance of telomere length occurs through the activity of the enzyme telomerase $;{ }^{27-29}$ however, enzymatic activity is not detected in most adult somatic cells. Telomere shortening has been demonstrated to induce a variety of genetic changes, including chromosome fusion at sites of shortened telomeres and recombination events, both of which contribute to genomic instability. ${ }^{17,18,30}$ Shortened telomeres have been identified in a variety of invasive cancers and preinvasive lesions of the pancreas and prostate, and telomere shortening may be a critical early event in the development of epithelial neoplasms. ${ }^{13,14,31,32}$ In addition to telomere shortening observed in dysplastic and carcinoma specimens, the majority of metaplastic lesions of the gallbladder in this study also demonstrated telomere shortening, supporting prior genetic evidence that metaplastic change in the gallbladder represents an early neoplastic alteration. ${ }^{7}$ Specifically, in the study of Wistuba et $a{ }^{7}{ }^{7}$ gallbladder metaplasias showed loss of heterozygosity in at least one cancer-associated locus in four of five cases. Perhaps the genetic instability promoted by telomere shortening promotes these genetic changes. Of note, in this study, we did not examine metaplastic lesions outside of the gallbladder. Metaplasia of the bile ducts has been studied infrequently, and we did not have adequate material to analyze it in this study. Metaplasia of the bile ducts has been associated with both inflammatory and neoplastic processes, and its role in neoplastic progression is not clear. ${ }^{33}$

The eventual outcome of telomere shortening includes such varied processes as apoptosis, cellular senescence, and genetic instability. ${ }^{16}$ The outcome of telomere shortening is influenced by additional genetic alterations within cells. For example, proper function of p53 is required to promote either senescence or apoptosis within cells carrying shortened telomeres, thus preventing proliferation of cells at high risk for neoplastic transformation. ${ }^{29,34,35}$ Mutations in the TP53 gene have been implicated in tumor progression, and these mutations are frequent in invasive biliary tract cancers. ${ }^{8}$ Interestingly, molecular alterations in TP53 have been identified in the majority of carcinoma in situ of the gallbladder, ${ }^{7,36}$ which suggests that shortened telomeres within this epithelium may promote tumor progression, rather than senescence or apoptosis.

Several of the infiltrating biliary adenocarcinomas and one case of carcinoma in situ demonstrated markedly heterogenous telomere lengths between cells (cell-cell heterogeneity). In addition, markedly increased telomere lengths were identified in one specimen of small cell carcinoma. These findings may be accounted for by varying rates of telomere shortening in individual cells, possible variable reactivation of telomerase within subpopulations of neoplastic cells, or utilization of the alternative 
lengthening telomeres pathway in a subset of biliary carcinomas. ${ }^{37,38}$

An important corroborative finding in these experiments is the normal telomere length present in inflamed but otherwise normal gallbladder epithelium. As carcinomas of the gallbladder typically arise in a background of chronic inflammation with metaplasia, lack of telomere length changes in non-metaplastic specimens suggests that telomere shortening is specific for longstanding pre-neoplastic and neoplastic changes, rather than acute, reactive changes associated with increased cell turnover. These findings are concordant with the clinical observation that only a small subset of patients with chronic cholecystitis is destined to develop carcinoma.

In summary, we demonstrate the reduction in telomere length in pre-neoplastic and neoplastic bilary tract epithelium, specifically in metaplastic and dysplastic gallbladder epithelium, as well as in invasive biliary tract carcinoma. This shortening appears specific for cells involved in or at risk for neoplastic transition and progression, rather than cells involved in regenerative or reactive processes.

\section{Acknowledgements}

This work was supported by the Margaret Lee Fund for Gallbladder and Bile Duct research, and the Mary M Graf and Gloria J Haen Endowment Funds for Gallbladder Cancer Research at The Johns Hopkins Hospital.

\section{References}

1 Landis SH, Murray T, Bolden S, et al. Cancer statistics. CA Cancer J Clin 1998;48:6-29.

2 Lazcano-Ponce EC, Miquel JF, Munoz N, et al. Epidemiology and molecular pathology of gallbladder cancer. CA Cancer J Clin 2001;51:349-364.

3 de Groen PC, Gores GJ, LaRusso NF, et al. Biliary tract cancers. N Engl J Med 1999;341:1368-1378.

4 Duarte I, Llanos O, Domke H, et al. Metaplasia and precursor lesions of gallbladder carcinoma. Frequency, distribution, and probability of detection in routine histologic samples. Cancer 1993;72:1878-1884.

5 Kamel D, Paakko P, Nuorva K, et al. p53 and c-erbB-2 protein expression in adenocarcinomas and epithelial dysplasias of the gall bladder. J Pathol 1993;170:67-72.

6 Tanno S, Obara T, Fujii T, et al. Proliferative potential and K-ras mutation in epithelial hyperplasia of the gallbladder in patients with anomalous pancreaticobiliary ductal union. Cancer 1998;83:267-275.

7 Wistuba II, Sugio K, Hung J, et al. Allele-specific mutations involved in the pathogenesis of endemic gallbladder carcinoma in Chile. Cancer Res 1995; 55:2511-2515.

8 Wistuba II, Albores-Saavedra J. Genetic abnormalities involved in the pathogenesis of gallbladder carcinoma. J Hepatobiliary Pancreat Surg 1999;6:237-244.

9 Itoi T, Watanabe H, Ajioka Y, et al. APC, K-ras codon 12 mutations and p53 gene expression in carcinoma and adenoma of the gall-bladder suggest two genetic pathways in gall-bladder carcinogenesis. Pathol Int 1996;46:333-340.

10 Blasco MA, Lee HW, Rizen M, et al. Mouse models for the study of telomerase. Ciba Found Symp 1997;211: 160-170; discussion 70-76.

11 Artandi SE, Chang S, Lee SL, et al. Telomere dysfunction promotes non-reciprocal translocations and epithelial cancers in mice. Nature 2000;406:641-645.

12 Meeker AK, Gage WR, Hicks JL, et al. Telomere length assessment in human archival tissues: combined telomere fluorescence in situ hybridization and immunostaining. Am J Pathol 2002;160:1259-1268.

13 van Heek NT, Meeker AK, Kern SE, et al. Telomere shortening is nearly universal in pancreatic intraepithelial neoplasia. Am J Pathol 2002;161:1541-1547.

14 Meeker AK, Hicks JL, Platz EA, et al. Telomere shortening is an early somatic DNA alteration in human prostate tumorigenesis. Cancer Res 2002;62:6405-6409.

15 Hackett JA, Greider CW. Balancing instability: dual roles for telomerase and telomere dysfunction in tumorigenesis. Oncogene 2002;21:619-626.

16 Kim SH, Kaminker P, Campisi J. Telomeres, aging and cancer: in search of a happy ending. Oncogene 2002; 21:503-511.

17 Blackburn EH. Telomere states and cell fates. Nature 2000;408:53-56.

18 Gasser SM. A sense of the end. Science 2000;288: 1377-1379.

19 Kim NW, Piatyszek MA, Prowse KR, et al. Specific association of human telomerase activity with immortal cells and cancer. Science 1994;266:2011-2015.

20 Shay JW, Bacchetti S. A survey of telomerase activity in human cancer. Eur J Cancer 1997;33:787-791.

21 Albores-Saavedra J, Henson DE, Klimstra DS. Tumors of the Gallbladder, Extrahepatic Bile Ducts, and Ampulla of Vater, Atlas of Tumor Pathology, 3rd series, Fascicle 27. Armed Forces Institute of Pathology: Washington, DC, p 39, 51.

22 Momoi H, Okabe H, Kamikawa T, et al. Comprehensive allelotyping of human intrahepatic cholangiocarcinoma. Clin Cancer Res 2001;7:2648-2655.

23 Wistuba II, Tang M, Maitra A, et al. Genome-wide allelotyping analysis reveals multiple sites of allelic loss in gallbladder carcinoma. Cancer Res 2001;61: 3795-3800.

24 Shiraishi K, Okita K, Kusano N, et al. A comparison of DNA copy number changes detected by comparative genomic hybridization in malignancies of the liver, biliary tract and pancreas. Oncology 2001;60:151-161.

25 Rijken AM, Hu J, Perlman EJ, et al. Genomic alterations in distal bile duct carcinoma by comparative genomic hybridization and karyotype analysis. Genes Chromosomes Cancer 1999;26:185-191.

26 von Zglinicki T. Oxidative stress shortens telomeres. Trends Biochem Sci 2002;27:339-344.

27 Prowse KR, Greider CW. Developmental and tissuespecific regulation of mouse telomerase and telomere length. Proc Natl Acad Sci USA 1995;92:4818-4822.

28 Holt SE, Wright WE, Shay JW. Multiple pathways for the regulation of telomerase activity. Eur J Cancer 1997;33:761-766.

29 Chiu CP, Harley CB. Replicative senescence and cell immortality: the role of telomeres and telomerase. Proc Soc Exp Biol Med 1997;214:99-106.

30 Hackett JA, Feldser DM, Greider CW. Telomere dysfunction increases mutation rate and genomic instability. Cell 2001;106:275-286. 
31 de Lange T, Shiue L, Myers RM, et al. Structure and variability of human chromosome ends. Mol Cell Biol 1990;10:518-527.

32 Hastie ND, Dempster M, Dunlop MG, et al. Telomere reduction in human colorectal carcinoma and with ageing. Nature 1991;350:866-868.

33 Hoang MP, Murakata LA, Padilla-Rodriguez AL, et al. Metaplastic lesions of the extrahepatic bile ducts: a morphologic and immunohistochemical study. Mod Pathol 2001;14:1119-1125.

34 Chin L, Artandi SE, Shen Q, et al. p53 deficiency rescues the adverse effects of telomere loss and cooperates with telomere dysfunction to accelerate carcinogenesis. Cell 1999;97:527-538.
35 Shay JW, Wright WE. Ageing and cancer: the telomere and telomerase connection. Novartis Found Symp 2001;235:116-125; discussion 25-29, 46-49.

36 Parwani A, Geradts J, Caspers E, et al. Immunohistochemical and genetic analysis of non-small cell and small cell gallbladder carcinoma and their precursor lesions. Mod Pathol 2003;16:299-308.

37 Bryan TM, Englezou A, Dalla-Pozza L, et al. Evidence for an alternative mechanism for maintaining telomere length in human tumors and tumor-derived cell lines. Nat Med 1997;3:1271-1274.

38 Le S, Moore JK, Haber JE, et al. RAD50 and RAD51 define two pathways that collaborate to maintain telomeres in the absence of telomerase. Genetics 1999;152:143-152. 